\title{
Limit Laws and Recurrence for the Planar Lorentz Process with Infinite Horizon
}

\author{
Domokos Szász Tamás Varjú
}

March 20, 2007

Dedicated to Ya. G. Sinai on the occasion of his seventieth birthday

\begin{abstract}
As Bleher, [B 92] observed the free flight vector of the planar, infinite horizon, periodic Lorentz process $\left\{S_{n} \mid n=0,1,2, \ldots\right\}$ belongs to the nonstandard domain of attraction of the Gaussian law - actually with the $\sqrt{n \log n}$ scaling. Our first aim is to establish his conjecture that, indeed, $\frac{S_{n}}{\sqrt{n \log n}}$ converges in distribution to the Gaussian law (a Global Limit Theorem). Here the recent method of Bálint and Gouëzel, [BG 06] helped us to essentially simplify the ideas of our earlier sketchy proof [SzV 04b]. Moreover, we can also derive a.) the local version of the Global Limit Theorem, b.) the recurrence of the planar, infinite horizon, periodic Lorentz process, and finally c.) the ergodicity of its infinite invariant measure.
\end{abstract}

Key words: Lorentz process, periodic configuration of scatterers, infinite horizon, corridors, non-normal domain of attraction of the Gaussian law, local limit law, recurrence, ergodicity

\section{Introduction}

The Lorentz process is the $\mathbb{Z}^{d}$-covering of a Sinai billiard - in other words of a dispersing billiard - given on $\mathbb{T}^{d}=\mathbb{R}^{d} / \mathbb{Z}^{d}$. If the horizon is finite, i. e. the free flight vector $\psi(x)$ is bounded, then the Lorentz process is a most instructive model of the Brownian motion.

We note here that though this should be true in any dimension $d \geq 2$, mathematical results only exist for $d=2$. In this paper we will also restrict our attention to this case. As suggested by the beautiful - partially rigorous, partially heuristic — work of Bleher [B 92], in the infinite horizon case $(d=2$ !) the asymptotic behaviour of the displacement $S_{n}$, taken in the moment of the $n^{\text {th }}$ reflection from a scatterer, is slightly superdiffusive and $\frac{S_{n}}{\sqrt{n \log n}}$ was expected to possess a limiting Gaussian distribution. The first main aim of the present work is to provide the first rigorous proof of this statement.

\footnotetext{
Research supported by the Hungarian National Foundation for Scientific Research grants No. T046187, NK 63066 and TS 049835, further by Hungarian Science and Technology Foundation grant No. A-9/03
} 
Theorem 1 (Global limit theorem). Suppose that the direction vectors of infinite, collision-free flights span the plane. Let $A$ be a bounded open set in the plane. Then

$$
\mu\left(\frac{S_{n}}{\sqrt{n \log n}} \in A\right) \rightarrow \int_{A} \phi_{\Sigma}(z) d z
$$

where $\phi$ is a nondegenerate Gaussian density with zero expectation and covariance matrix $\Sigma$.

A mostly geometric proof of this theorem was sketched in [SzV 04b]. However, before we had completed our paper with the technical proof of Theorem 1, there appeared a much interesting work of Bálint and Gouëzel, [BG 06]: for the stadium billiard they gave a quite analytic proof of a global limit theorem which also uses the $\sqrt{n \log n}$ scaling. The coincidence of scalings is explained by the analogous behaviour of long free flights in our model (i. e. in corridors of the Lorentz process) and that of the quasi integrable trajectories between the linear sides of the stadium billiard (the neighbourhood of bouncing ball orbits). The arguments of [BG 06] also helped us to simplify our approach substantially at essentially three points:

1. once one has a tower construction à la Young, their Lemma 3.5 (cf. our Theorem 13) provides a general, concise condition for the validity of a non-standard Gaussian limit law;

2. they reduce the "tower-sums" to a more tractable, still dominant part;

3. for describing excursions from the tail they use a delicate result of Chernov, [Ch 99] (see in our Lemma 16).

A well-known spectacular property of discrete approximations of the Brownian motion, like of simple symmetric random walks, is Pólya's classical theorem, claiming recurrence in the planar case.

For the diffusive, finite horizon case of the Lorentz process this had been proved by various authors in [Sch 98], [Con 99], [SzV 04a]. (As a matter of fact, the problem was raised by Sinai in 1979, after he, with Bunimovich, had proved its convergence to the Brownian motion. In 1985 - as a partial solution the first named author jointly with A. Krámli could settle a weaker property: quasi-recurrence of the planar, finite horizon Lorentz process, cf. [KSz 85].)

The arguments of [SzV 04a] relied on a local version, LCLT of the central limit theorem (CLT). Our second main aim here is to first deduce a local limit theorem (Theorem 2, a LLT), a local version of Theorem 1 and then by using it to establish the recurrence of the planar Lorentz process with an infinite horizon (Theorem 3).

Theorem 2 (Local limit theorem). Suppose that the direction vectors of infinite, collision-free flights span the plane. Let $k_{n} \in \mathbb{Z}^{2}$ such that $\frac{k_{n}}{\sqrt{n \log n}} \rightarrow k \in \mathbb{R}^{2}$. Then for the discretised position vector $S_{n}^{\text {disc }}$ the following holds:

$$
n \log n \mu\left(S_{n}^{\mathrm{disc}}=k_{n}\right) \rightarrow \phi_{\Sigma}(k)
$$

where $\phi_{\Sigma}$ is a nondegenerate Gaussian density with zero expectation from Theorem 1. 
Theorem 3 (Recurrence).

$$
\mu\left(\exists n_{k} \rightarrow \infty \quad S_{n_{k}}^{\text {disc }}=0\right)=1
$$

It is worth mentioning that, for the planar Lorentz process with a finite horizon but with a finite modification of a periodic scatterer configuration, the problem of recurrence and of ergodicity was studied by Lenci [L 03].

The recurrence itself also has an additional interesting conclusion. Let us note first that for the Lorentz process strong stochastic properties, like correlation decay, limit laws, etc. could only be obtained in the case of a periodic configuration of scatterers for then its factor is a Sinai billiard. For this same case, however, it is an interesting question whether the Lorentz dynamics is ergodic without this factorisation as well (N. B. in this case the invariant measure is infinite!). The combination of Theorem 3 with an old result of Simányi, [Sim 89] also provides the answer:

Theorem 4. The planar Lorentz dynamics with a periodic configuration of scatterers is ergodic.

Theorems 3 and 4 are true in the finite horizon case also (see above).

The paper is organised as follows. Section 2 is devoted to the description of the model and to the study of its important geometric properties. In fact, we recall the notion of corridors also discussed in Bleher's work; they are distinguished parts of the phase space in whose neighbourhood the free flight vector becomes unbounded. Here we also introduce new coordinates to make easier the study of the tail behaviour of the free flight vector and its asymptotic Markov property. In particular, our geometric probabilistic estimates are sharper than those of [B 92]. Finally Section 2 closes with a brief reminder about the nonnormal domain attraction of the normal law. Section 3 is preparatory to the technique used: it contains a reminder on Young towers and on the Fourier transform of the Perron-Frobenius operator. Section 4 contains the proof of Theorem 1, while section 5 those of Theorems 2, 3 and 4 .

For the convenience of the reader we summarise the main results used from other papers:

- Young's tower construction which, in fact, is briefly recalled in section 3.1;

- The singularity structure (recalled in section 2.3) and the growth lemma (used in the proof of lemma 16) from Chernov [Ch 99] (or alternatively from Chapter 5 of [ChM 06]);

- Theorem 13 and Lemma 15 of this paper from [BG 06].

\section{The model and its geometry}

\subsection{The model}

Consider finitely many scatterers $\mathcal{O}_{i}$ on the 2 -torus, (also called obstacles) $\mathbb{T}^{2} \supset$ $\mathcal{O}=\cup \mathcal{O}_{i}$ such that each of the scatterers is strictly convex with a $\mathcal{C}^{3}$-smooth boundary. Let $n(q)$ denote the unit normal vector of the boundary $\partial \mathcal{O}$ at the point $q$, directed outwards $\mathcal{O}$. The phase space of the system is:

$$
X=\left\{\left(q \in \partial \mathcal{O}, v \in \mathbb{R}^{2}\right)|| v \mid=1,\langle v, n(q)\rangle \geq 0\right\} .
$$


The dynamics $T: X \rightarrow X$ is uniform motion with $v$ velocity vector followed by an elastic collision ( $v$ is mirrored to the tangent line at the point of impact). This system has a natural invariant measure: if we denote by $l$ the total length of $\partial \mathcal{O}$, then $d \mu=\frac{1}{2 l}\langle v, n(q)\rangle d q d v$, is an invariant probability measure, since $\int_{X}\langle v, n(q)\rangle d q d v=2 l$. The normalising constant $\frac{1}{2 l}$ will be denoted by $c_{\mu}$.

This phase space will be identified with a finite number of cylinders $\partial \mathcal{O} \times$ $\left[-\frac{\pi}{2} ; \frac{\pi}{2}\right]$. So in this paper if $v$ denotes a velocity of a phase point it is meant as $v \in\left[-\frac{\pi}{2} ; \frac{\pi}{2}\right]$.

The boundary of this phase space consists of tangential collisions denoted by $\mathcal{S}_{0}$. The dynamics resp. the inverse dynamics is non-continuous in backward resp. forward images of this set. We will denote $\mathcal{S}_{i}=T^{i} \mathcal{S}_{0}, i \in \mathbb{Z}$.

The planar Lorentz process is the natural $\mathbb{Z}^{2}$ cover of the above-described toric billiard. More precisely: consider $\Pi: \mathbb{R}^{2} \rightarrow \mathbb{T}^{2}$ the factorisation by $\mathbb{Z}^{2}$. Its fundamental domain $D$ is a square (semi-open, semi-closed) in $\mathbb{R}^{2}$, so $\mathbb{R}^{2}=$ $\cup_{z \in \mathbb{Z}^{2}}(D+z)$, where $D+z$ is the translated fundamental domain.

We lift the obstacles to the plane (i. e. we take $\tilde{\mathcal{O}}=\Pi^{-1} \mathcal{O}$ ), and define the phase space $\tilde{X}$, and the dynamics $\tilde{T}$ exactly the same way as above. The free flight function $\tilde{\psi}: \tilde{X} \rightarrow \mathbb{R}^{2}$ is defined as follows: $\tilde{\psi}(\tilde{x})=\tilde{q}(\tilde{T} \tilde{x})-\tilde{q}(\tilde{x})$. The discrete free flight function $\tilde{\kappa}: \tilde{X} \rightarrow \mathbb{Z}^{2}$ is defined as follows: $\tilde{\kappa}(\tilde{x})=\iota(\tilde{T} \tilde{x})-\iota(\tilde{x})$, where $\iota(\tilde{x})=z$ if $q(\tilde{x}) \in D+z$. Observe finally that $\tilde{\psi}$ and $\tilde{\kappa}$ are invariant under the $\mathbb{Z}^{2}$ action, so there are $\psi$ and $\kappa$ functions defined on $X$, such that $\tilde{\psi}=\Pi^{*} \psi$ and $\tilde{\kappa}=\Pi^{*} \kappa$. Actually for our purposes it will be more convenient to choose the fundamental domain in such a way that $\partial \tilde{\mathcal{O}} \cap \partial D=\emptyset$. In this way $\kappa$ will be continuous.

Definition 5. The system is said to have finite horizon if the free flight function is bounded. Otherwise the system is said to have infinite horizon.

\subsection{The corridors, their geometry and the tail of the free flight}

¿From now on we will be only considering the infinite horizon case. It had been observed in the physical literature (cf. [FM 84], [BD 85], [Bun 85] and [ZGNR 86]) that, in this case, the anomalous diffusive behaviour is intimately related to the unboundedness of the free flight vector. More precisely it is related to its tail behaviour that is to the behaviour of the free flight vector in the neighbourhood of collision-free orbits. This motivated Bleher [B 92] to introduce corridors and to study probabilistic geometric estimates of the tail behaviour of free flight vectors in these corridors. Our method, however, requires more delicate estimates (the results are also sharper), and therefore we are starting to build up our language.

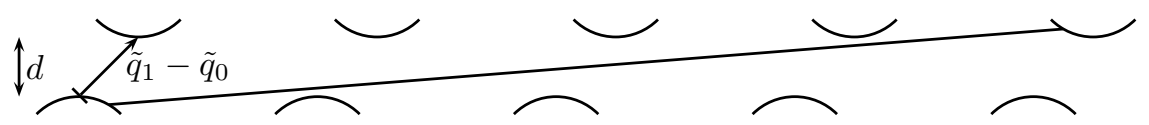

Figure 1: Free-flight crossing a corridor, and some geometric constants

In the infinite horizon case the only reason for the unboundedness of the free flight is the presence of corridors. These are bi-infinite strips in the billiard-table $\mathbb{R}^{2} \backslash \tilde{\mathcal{O}}$. The strips are tangent to the obstacles, and their slope is necessarily 


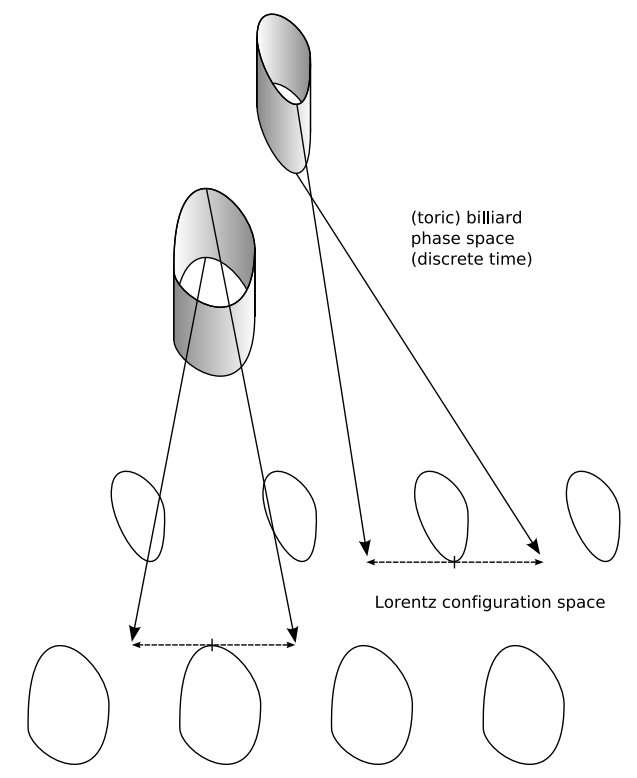

Figure 2: A corridor, and the corresponding four phase points.

rational, and, moreover, - up to $\mathbb{Z}^{2}$ translations - there are finitely many of them. We will suppose the - geometrically generic - condition: for each corridor, and each side of the corridor the tangent obstacles are the images of a single scatterer under $\mathbb{Z}^{2}$ translations. Our results are also valid in the excluded cases, but the geometric constants, which we will calculate would have a more complicated form.

For such a corridor there are four corresponding points in the phase-space, as shown on figure 2 . These points are on the boundary of $X$, and are fixed by the dynamics. (Without the previous condition these would be only periodic points.) Outside of the neighbourhood of these points the free-flight is bounded.

Let us fix such a fixed point on the boundary as $x_{0}=\left(q_{0} ; v_{0}\right)$, where $v_{0}$ is either $\pi / 2$ or $-\pi / 2$. Let us denote by $\mathcal{O}_{0}$ the obstacle on which $q_{0}$ is placed. The free flight $\psi\left(x_{0}\right)$ is a lattice vector $\psi\left(x_{0}\right)=\kappa\left(x_{0}\right)$, since $T x_{0}=x_{0}$. Denote $\kappa\left(x_{0}\right)=w_{0}$, and the curvature of $\mathcal{O}_{0}$ at $q_{0}$ by $\xi_{0}$. Denote the considered (small enough) neighbourhood of $x_{0}$ by $U_{0}$.

In $U_{0}$ (means close enough to $x_{0}$ ) two types of nonsingular collisions can happen. First when the moving particle is "crossing" the corridor (see figure 1). In this case the free-flight is long (actually this is the only case), the closer the phase point lies to $x_{0}$ the longer the free-flight can be. The next collision happens on the "other side" of the corridor. To make it precise let us denote by $x_{1}=\left(q_{1} ; v_{1}\right)$ the phase point which corresponds to the same corridor as $x_{0}$, but $v_{1}=-v_{0}$ and $q_{1} \neq q_{0}$ (see figure 2 ). Denote by $\vec{Q}_{0}$ the planar lattice vector $\iota\left(\tilde{q}_{1}\right)-\iota\left(\tilde{q}_{0}\right)$, where the lifting is such that $\tilde{q}_{1}-\tilde{q}_{0}$ "crosses" the corridor (see figure 1). We also need to define the "width" of the corridor $d$ which is the length of that component of $\tilde{q}_{1}-\tilde{q}_{0}$ which is perpendicular to $w_{0}$.

The other type of nonsingular trajectory in $U_{0}$ is when the next collision is on the "same side" of the corridor i. e. $\kappa=w_{0}$. The phase point $T x$ is then again close to $x_{0}$, but this time it is of the first type, so consecutive "same side" 


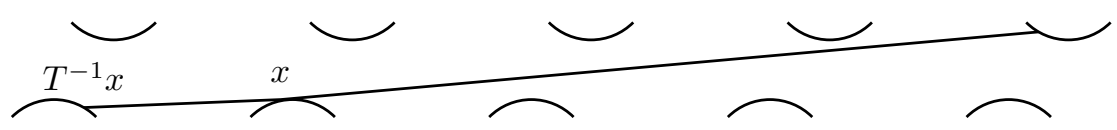

Figure 3: If phase point $T^{-1} x$ is such that $\kappa(x)=w_{0}$, then if $x \in U_{0}$ the free flight starting in $q(x)$ "crosses" the corridor.

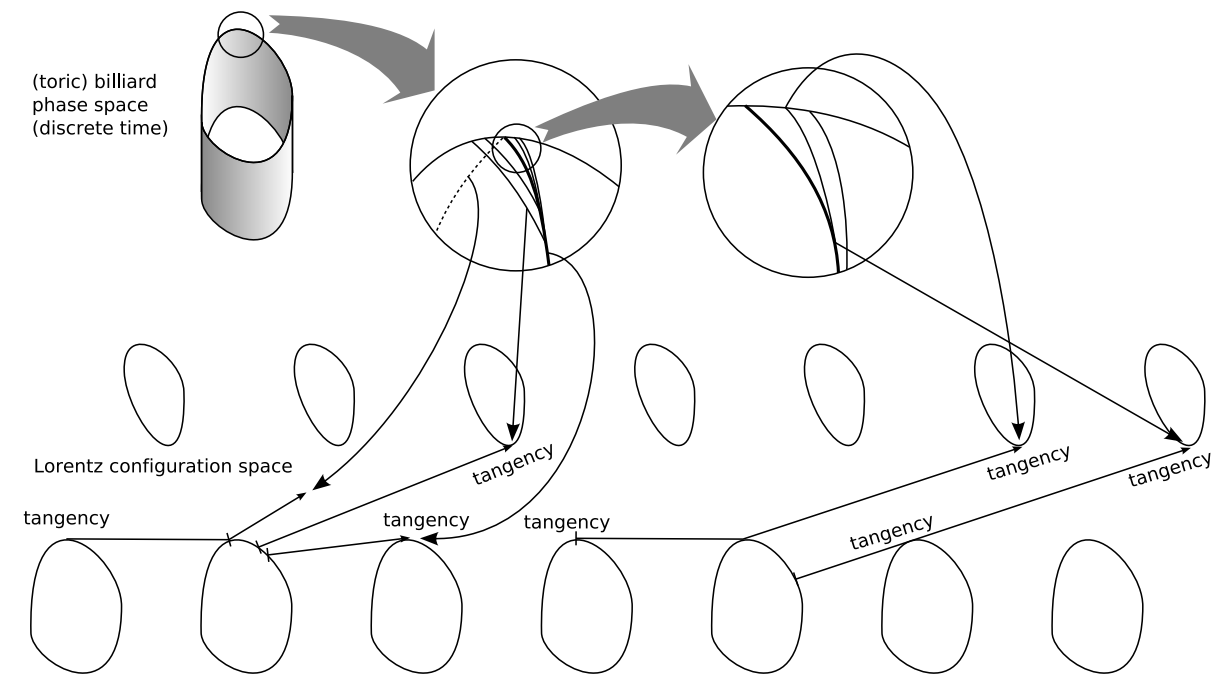

Figure 4: Singularity structure and singular trajectories near $x_{0}$

collisions cannot happen. This can be seen on figure 3 .

Proposition 6. Let $U_{0} \subset X$ be a sufficiently small neighbourhood of $x_{0}$ then

$$
\mu\left\{x \in U_{0} \mid \kappa(x)=N w_{0}+\vec{Q}_{0}\right\} \sim c_{\mu} d^{2}\left|w_{0}\right|^{-1} N^{-3}
$$

The range of $\kappa$ in $U_{0}$ is $\vec{Q}_{0}+w_{0} \mathbb{Z}^{+}$with possibly finitely many exceptions.

We postpone the sketchy proof for the first fact to section 2.4. The second statement was essentially proved in the above text.

\subsection{The singularity structure}

We are going to describe the singularity structure, and the type of singular trajectories in $U_{0}$. The importance of this is that singularities bound the sets for which we want to derive measure estimates. On figure 4 we also plot some trajectory-segments, the configuration component of the corresponding phase point is denoted by a small tick perpendicular to the trajectory.

There is a singularity curve from $\mathcal{S}_{-1}$ which is a preimage of tangential collisions, denoted by thick line in figure 4 , starting from $x_{0}$. This consists of phase points where the next collision will be tangential on $\mathcal{O}_{0}+w_{0}$. This is called the "main" singularity. The reason for this is not that it would be more singular, but rather the fact that other singularities end on this line.

There are singularity curves printed on the left of this one, starting on the boundary of the phase space and ending on the main singularity. These consist of 


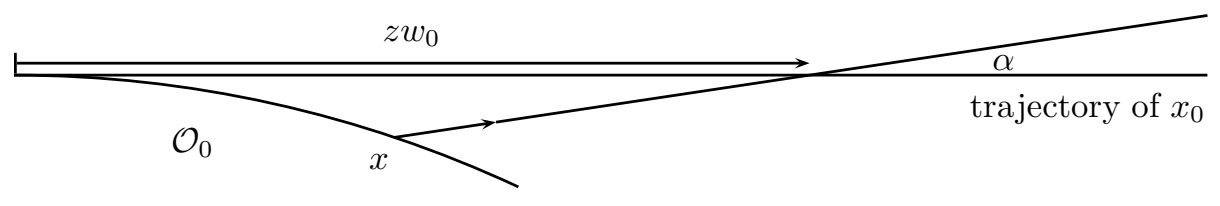

Figure 5: The new coordinates $z, \alpha$.

phase points where the free flight "crosses" the corridor, and the next collision is tangential. Therefore this is also a part of $\mathcal{S}_{-1}$. A level-set of the discretised freeflight function $\kappa$ consists of a curvilinear rectangle bounded by two neighbouring curves from this singularity family, the edge of the phase space and the main singularity.

On the right of the main curve there are some curves from $\mathcal{S}_{-2}$. These also start on the boundary and end on the main curve, but unlike the previous ones, these curves have zero angle with the main line. These consist of phase points, for which the first collision is on $\mathcal{O}_{0}+w_{0}$, and the next one is tangential after crossing the corridor. On the right of the main singularity the next collision for any phase point occurs on $\mathcal{O}_{0}+w_{0}$, therefore $\kappa$ is constant $w_{0}$ in this half of $U_{0}$.

These two families of singularity curves have an infinite number of pieces accumulating in $x_{0}$. The closer the curve is to $x_{0}$ the further the tangential collision occurs, after the moving particle has crossed the corridor. The main singularity curve has slope $-\xi_{0}$ on the boundary of the phase space. For the other singularity curves the slope on the boundary of the phase space is asymptotically $-\xi_{0}$ as they approach $x_{0}$.

The last singularity curve we want to describe is from $\mathcal{S}_{1}$. It consists of phase points, where the previous collision was tangential on $\mathcal{O}_{0}-w_{0}$. This is the image of the half of $\partial X \cap U_{0}$, namely that half where $\kappa=w_{0}$ (on the right of $x_{0}$ on figure 4 ). Consequently (since $x_{0}$ is fixed) this curve starts at $x_{0}$. This is drawn with a dotted line on figure 4 . This curve has slope $\xi_{0}$ on the boundary. The sign of the second derivatives of all the singularity curves can be read from the picture.

\subsection{New coordinates, and the joint distribution of $\kappa, \kappa \circ T^{-1}$}

In this subsection some proofs will be omitted, some will be sketchy or require further estimates. However, since the missing parts rely on simple but tedious geometrical calculations and the application of these results in later sections does not need sharp estimates, we intended to keep this section not too long.

Instead of using the original $\left(q_{0}, v_{0}\right)$ coordinates we are going to introduce new $(\alpha, z)$ coordinates in $U_{0}$. The new coordinates $z$ and $\alpha$ are shown on figure 5 (these coordinates are also different from those of [B 92]). During the freeflight, bouncing off the scatterer $\mathcal{O}_{0}$, the trajectory of $x$ crosses the trajectory of $x_{0}$. This crossing point is therefore $q_{0}+z w_{0}$ for some $z \in \mathbb{R}$. The reader should convince himself that the coordinate $z$ is, in fact, a periodic one with period 1 . The other coordinate $\alpha$ is the angle $\measuredangle\left(w_{0}, \psi(x)\right)$.

The reason for this change is that these coordinates are more suitable for computations in relation with the free-flight since they are more intrinsically related to the geometry of the model, especially asymptotically (when the free flight goes to infinity). For example, the free flight has the asymptotic form 
$|\kappa| \sim \frac{d}{\alpha}$ where $d$ is the 'width' of the corridor (cf. fig. 1). Also, the invariant measure is asymptotically equal to $c_{\mu}|\alpha|\left|w_{0}\right| d z d \alpha$.

We note that the crossing point (which was the base of this coordinatisation) does not exist when the next collision occurs on $\mathcal{O}_{0}+w_{0}$. So these new coordinates map only the half of $U_{0}$ to the $(z, \alpha)$ plane. Namely that half which is drawn on the left of the main singularity on figure 4 . We will denote this part by $U_{0}^{\prime}$ (remember that $x_{0}$ and $U_{0}$ are fixed). However, this restriction does not influence the study of asymptotics, since we miss only the $w_{0}$-level set of $\kappa$ inside $U_{0}$.

Proof of Proposition 6. The level-set of $\kappa$ is a curvilinear rectangle in the $(z, \alpha)$ plane (cf. figure 6). We are going to multiply the height, the width and the density to get the measure. The width is simply 1 . The height can be obtained from the formula $\alpha \sim \frac{d}{|\kappa|}$. Writing $\alpha^{\prime} \sim \frac{d}{|\kappa|+\left|w_{0}\right|}$ we get $\alpha-\alpha^{\prime} \sim \frac{d\left|w_{0}\right|}{|\kappa|^{2}}$. The density is $c_{\mu}|\alpha|\left|w_{0}\right|$ and by substituting $\alpha$ we get $c_{\mu} d \frac{\left|w_{0}\right|}{|\kappa|}$. So the measure is $\sim \frac{c_{\mu} d^{2}\left|w_{0}\right|^{2}}{|\kappa|^{3}}$.

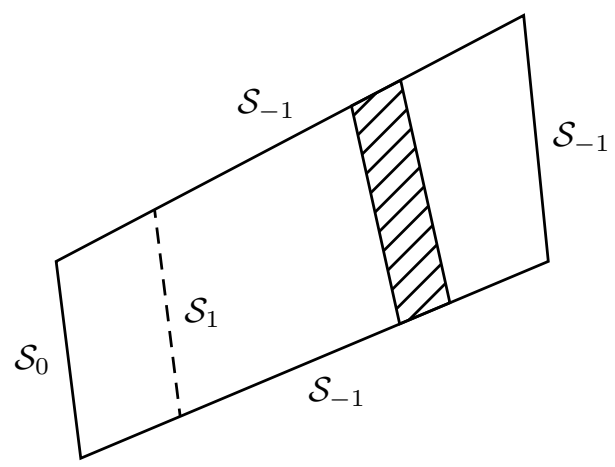

Figure 6: Level set of $\kappa$ inside $U_{0}$, and its intersection with a level set of $\kappa \circ T^{-1}$ (in the $(z, \alpha)$ coordinate plane)

Let us explain how this $(z, \alpha)$ image on figure 6 is related to the phase portrait on figure 4 explained before. The largest curvilinear rectangle on figure 6 is the image of a level-set of $\kappa$ under the $(z, \alpha)$ coordinate-mapping. This level set is bounded by the boundary of the phase-space on the left, two singularity curves from the first family on the top, and on the bottom, and the main singularity on the right. There is also the dashed line, which is the singularity line from $\mathcal{S}_{1}$, already explained before, too.

This latter line plays an important role in the joint distribution. On the left of this line $\kappa \circ T^{-1}=w_{0}$. On the right of this line the mapping $T^{-1}$ takes values in the neighbourhood of another corridor-phase-point $x_{1}$ (see subsection 2.2). We are going to use $w_{1}, \xi_{1}, U_{1}, U_{1}^{\prime}$ for the point $x_{1}$ in the same sense as $w_{0}, \xi_{0}, U_{0}, U_{0}^{\prime}$ have been used for the point $x_{0}$. Using this notation, on the right of the line we are describing now, there lies $T U_{1}^{\prime}$.

Talking about the joint distribution in terms of our new coordinate functions, note that the $\alpha$ coordinate function is defined in $U_{0}^{\prime}$. Therefore $\alpha \circ T^{-1}$ in the 
domain $U_{0}^{\prime} \cap T U_{1}^{\prime}$ has to be meant as applying the same coordinatisation rule in $U_{1}$. Since $w_{1}=w_{0}$, the $\alpha$ coordinate functions in $U_{0}$ and $U_{1}$ are comparable also as absolute angles: the observer only has to change signs.

By definition the sign of $\alpha$ is positive, and $z$ is mostly positive. More precisely remember that the asymptotic form of the invariant measure does not depend on $z$ meaning that in the $|\kappa| \rightarrow \infty$ limit the distribution of $z$ is uniform. Now consider the range of $z$ in the domain $\kappa=\vec{N}$ :

$$
z_{\min } \stackrel{\text { def }}{=} \min \left\{z(x) \mid x \in U_{0}, \quad \kappa(x)=\vec{N}\right\}
$$

and respectively

$$
z_{\text {max }} \stackrel{\text { def }}{=} \max \left\{z(x) \mid x \in U_{0}, \quad \kappa(x)=\vec{N}\right\}
$$

Proposition 7. The asymptotics of the range of $z$ in the domain $\kappa=\vec{N}$ when $\vec{N}$ is in the range of $\kappa$ (see proposition 6) and $|\vec{N}| \rightarrow \infty$ is

$$
z_{\min } \sim 1-z_{\max } \sim \frac{d}{2\left|w_{0}\right| \xi_{0}|\vec{N}|}
$$

Easy geometrical calculations yield the collision equation:

Proposition 8. $O n U_{0}^{\prime} \cap T U_{1}^{\prime}$ :

$$
\alpha \circ T^{-1} \sim-\alpha+2 \sqrt{\alpha^{2}+2 \alpha z\left|w_{0}\right| \xi_{0}} \quad(\alpha \rightarrow 0)
$$

Proposition 9. In $U_{0}$ the following holds:

$$
\min |\kappa| \circ T^{-1} \sim \sqrt{\frac{d|\kappa|}{8 \xi_{0}\left|w_{0}\right|}} \quad \text { and } \max |\kappa| \circ T^{-1} \sim \frac{8 \xi_{0}\left|w_{0}\right||\kappa|^{2}}{d} \quad|\kappa| \rightarrow \infty
$$

Proof. Substituting the asymptotic maximum of $z(\max z \rightarrow 1$ as $\alpha \rightarrow 0)$ to the collision equation and omitting non-dominant terms we get $\max \alpha \circ T^{-1} \sim$ $2 \sqrt{2 \alpha\left|w_{0}\right| \xi_{0}}$. Since $\alpha \sim \frac{d}{|\kappa|}$ and $\alpha \circ T^{-1} \sim \frac{d}{\left|\kappa \circ T^{-1}\right|}$, substituting $\alpha$ and $\alpha \circ T^{-1}$ and then rearranging yields the first statement of the proposition. Using the time-reversion symmetry for this formula we get the second one.

We can also compute the joint distribution of $\left(\kappa, \kappa \circ T^{-1}\right)$.

\section{Proposition 10.}

$$
\mu\left(\{\kappa=\vec{N}\} \cap\left\{\kappa \circ T^{-1}=\vec{M}\right\} \cap U_{0}\right) \lesssim \frac{c_{\mu} d^{3}\left|w_{0}\right|^{2}}{4 \xi_{0}} \frac{|\vec{N}|+|\vec{M}|}{|\vec{N}|^{3}|\vec{M}|^{3}}
$$

Proof. For a nonempty intersection we are going to multiply the height the width and the density to get the measure. The density and the height are the same as in the proof of proposition 6 . For the width consider the derivative of the collision equation

$$
\frac{\partial \alpha \circ T^{-1}}{\partial z} \sim \frac{2 \alpha\left|w_{0}\right| \xi_{0}}{\sqrt{\alpha^{2}+2 \alpha z\left|w_{0}\right| \xi_{0}}}
$$


To express the square root in terms of $\kappa$ and $\kappa \circ T^{-1}$ we can rearrange the collision equation, and substitute $\alpha$ and $\alpha \circ T^{-1}$ :

$$
\frac{d}{\left|\kappa \circ T^{-1}\right|}+\frac{d}{|\kappa|} \sim 2 \sqrt{\alpha^{2}+2 \alpha z\left|w_{0}\right| \xi_{0}}
$$

We can express the increment of $z$ as the inverse of the derivative multiplied by the increment of $\alpha \circ T^{-1}$. That is

$$
\frac{\frac{d}{\left|\kappa \circ T^{-1}\right|}+\frac{d}{|\kappa|}}{4 \frac{d}{\kappa}\left|w_{0}\right| \xi_{0}} \frac{d\left|w_{0}\right|}{\left(\kappa \circ T^{-1}\right)^{2}} \sim d \frac{\kappa+\kappa \circ T^{-1}}{4 \xi_{0}\left(\kappa \circ T^{-1}\right)^{3}}
$$

Hence the proposition.

The measure of the set $\{\kappa=\vec{N}\} \cap\left\{\kappa \circ T^{-1}=\vec{M}\right\} \cap U_{0}$ can be zero when $\vec{N}$ or $\vec{M}$ is not in the range of $\kappa$ inside $U_{0}$ (see proposition 6 ) or they fail the range inequality (we only gave the asymptotics of this in proposition 9 roughly $\left.c_{3} \sqrt{|\vec{N}|}<|\vec{M}|<c_{4}|\vec{N}|^{2}\right)$. It can be also smaller than the expression given in proposition 10 when the pair $(\vec{N}, \vec{M})$ is close to the boundary of the range inequality, but we do not want to formulate the validity precisely, we just mention that in most part of the domain the inequality is sharp. It can also be checked by summing the right hand side, and getting 1 in the limit.

What this essentially means is that the previous free-flight $\left|\kappa \circ T^{-1}\right|$ is mostly in the range of $\sqrt{|\kappa|}$. The measure of being in any other range can be estimated from above with $|\kappa|$ powers. To formulate precisely what we will use later in subsection 4.1:

\section{Proposition 11.}

$$
\mu\left(\{\kappa=\vec{N}\} \cap\left\{\kappa \circ T^{-1}>|\kappa|^{\frac{3}{4}}\right\} \cap U_{0}\right)=O\left(|\vec{N}|^{-3.5}\right)
$$

Proof. $\sum_{M=N^{\frac{3}{4}}} \frac{N+M}{N^{3} M^{3}}=O\left(N^{-3.5}\right)$

The other level set of $\kappa \circ T^{-1}$ which intersects $U_{0}$ is the $\kappa \circ T^{-1}=w_{0}$ set.

\section{Proposition 12.}

$$
\mu\left(\{\kappa=\vec{N}\} \cap\left\{\kappa \circ T^{-1}=w_{0}\right\} \cap U_{0}\right)=O\left(|\vec{N}|^{-4}\right)
$$

Proof. As before the measure will be estimated with the product of the height, density and the width. It remained to estimate only the width. The domain is the left-hand side of the dashed line on figure 6 . Going from right to left inside the level set in figure 6 , the dashed line is reached exactly when $\alpha \circ T^{-1}$ reaches its minimum. According to proposition 9 we have to estimate what is the value of $z$ for which $\alpha \circ T^{-1}$ reaches const $\cdot \alpha^{2}$. Exact calculations based on the derivative of the mapping would give $O\left(|\vec{N}|^{-5}\right)$ in the right hand side of the proposition, but here it is sufficient to observe that this singularity line denoted by the dashed curve is on the left of the $z=0$ line, where $\alpha=\alpha \circ T^{-1}$. So the width can be estimated by $z_{\min }$ which was given in proposition 7 . 
Lastly we have to introduce homogeneity strips. This is a traditional tool in the theory of hyperbolic billiards to ensure the necessary distorsion bounds (cf. [ChM 06]).

$$
\mathbb{H}_{k}=\left\{(q, v) \in X \mid \pi / 2-k^{-2}<v<\pi / 2-(k+1)^{-2}\right\}
$$

and

$$
\mathbb{H}_{-k}=\left\{(q, v) \in X \mid-\pi / 2+(k+1)^{-2}<v<-\pi / 2+k^{-2}\right\}
$$

for all $k \geq k_{0}$ and

$$
\mathbb{H}_{0}=\left\{(q, v) \in X \mid-\pi / 2+k_{0}^{-2}<v<\pi / 2-k_{0}^{-2}\right\}
$$

where $k_{0}$ is a fixed, suitably large constant.

These strips can also be expressed in the terms of $\kappa$ and $\kappa \circ T^{-1}$ since the distance from the boundary of the phase-space in $U_{0}^{\prime} \cap T U_{1}^{\prime}$ is asymptotically:

$$
\frac{\frac{d}{|\kappa|}+\frac{d}{\left|\kappa \circ T^{-1}\right|}}{2} \text {. }
$$

It follows that the smallest $k$ index such that $\mathbb{H}_{k}$ intersects $\{|\kappa|=N\}$ is: $\max \left\{k_{0}, c N^{1 / 4}\right\}$.

\subsection{Non-normal domain of attraction of the normal law}

First we summarize the necessary information about domains of attraction focusing mainly on our interest: the non-normal domain of attraction of the normal law (cf. the monographs [IL 71], section II/6 or [F 66], section XVII/5). A random variable $R$ with distribution function $\mathbb{P}_{R}$ belongs to the domain of attraction of a normal distribution if its characteristic function satisfies

$$
\log \int e^{i t u} d \mathbb{P}_{R}(u)=i t \nu-\frac{1}{2} t^{2} L(1 /|t|)(1+o(1)) \quad(t \rightarrow 0)
$$

for some constant $\nu \in \mathbb{R}$ and a slowly varying function $L:(0, \infty) \rightarrow(0, \infty)$ which is bounded below. (Note that $o(1)$ can be complex.) Recall that the positive function $L:(0, \infty) \rightarrow(0, \infty)$ is slowly varying at infinity if for each $x>0$ as $t \rightarrow \infty$

$$
\frac{L(t x)}{L(t)} \rightarrow 1
$$

(cf. [F 66] VIII/8).

It is worth mentioning that the slowly varying function in the above equation can be chosen as

$$
L(x)=\int_{-x}^{x} u^{2} d \mathbb{P}_{R}(u)
$$

In the sequel we fix $L$ in this way and thus it will, of course, be a monotone function. In general, normal domains of attractions of stable laws are characterised by the fact that in the limit theorems formulated for them the scaling has an exact polynomial form. The distribution function $\mathbb{P}_{R}$ belongs to the normal domain of attraction of the normal law if and only if $L$ is bounded. Our interest here, however, is its non-normal domain of attraction. Denote $\chi(x)=1-F(x)+F(-x)$. Then $\mathbb{P}_{R}$ belongs to the non-normal domain of the gaussian law if and only if one of the following conditions hold: 
- $L(x)=\int_{-x}^{x} u^{2} d \mathbb{P}_{R}(u)$ is an unbounded slowly varying function;

- $\chi(x)=\frac{l(x)}{x^{2}}$ where $l(x)$ is a slowly varying function; if so, then it is true that $l(x)=o(L(x))$.

Finally, for a random variable $R$ in the non-normal domain of attraction of the normal law, the independent sum of $\mathbb{P}_{R}$-distributed random variables $S_{n}^{*}$ satisfies the limit theorem: $\frac{S_{n}^{*}-n \nu}{B_{n}} \stackrel{\mathrm{d}}{\rightarrow} \mathcal{N}(0,1)$ as $n \rightarrow \infty$ in distribution, where $B_{n}$ is the normalising sequence defined by the asymptotics $\frac{n L\left(B_{n}\right)}{B_{n}^{2}} \rightarrow 1$.

Let us turn now to our situation. The random variable $\kappa$ is a vector. It was shown in proposition 6 that its component function belongs to the non-normal domain of attraction of the normal law, if that component is not perpendicular to all of the corridor-free-flights $w_{i}$. In this case $l(x)$ is a constant function $l(x) \equiv \mathbf{c}$. Since the free-flight is symmetric, we have $c_{1}=c_{2}=\frac{1}{2}$, and $\nu=0$. Consequently $L(x) \sim 2 \mathbf{c} \log x$, and $B_{n}=\sqrt{\mathbf{c} n \log n}$ is a normalising sequence. The constant $\mathbf{c}$ depends on which component we are looking at. If we choose $\vec{v} \in \mathbb{R}^{2}$ a unit vector, then the constant of the $\vec{v}$ component has the following expression in the terms of the geometric constants:

$$
\mathbf{c}=\sum_{x \in \partial X \mid T x=x} c_{\mu} d_{x}^{2} \frac{\langle\psi(x), \vec{v}\rangle^{2}}{2|\psi(x)|} .
$$

Remember that for such points $\psi(x)=\kappa(x)$, and we used the notation $\psi\left(x_{0}\right)=$ $w_{0}$ for the fixed corridor we were investigating. Also note, that every term in the above sum appears exactly four times (cf. figure 2 in section 2.2)!

Since the configuration is planar, the following is true: if the corridor freeflight vectors span the plane, then every component of $\kappa$, hence the vector itself is in the non-normal domain. If this is not the case, then one has to apply anisotropic scaling to get a nondegenerate limit distribution. Namely it should be $\left(\begin{array}{cc}\sqrt{n} & 0 \\ 0 & \sqrt{n \log n}\end{array}\right)$ in a basis, where the first element is perpendicular to the corridor free-flights.

The goal of the forthcoming arguments is to establish Bleher's hypothesis: though the (stationary) process of the free flights of our model is not an independent process, nevertheless in many respects the partial sums behave asymptotically the same way as if the variables were independent.

\section{Young towers and the Fourier transform of the Perron-Frobenius operator}

\subsection{Young towers}

According to our recent understanding the most efficient way for constructing Markov partitions for billiards is to use Young towers, cf. [You 98]. We are going to introduce the main concepts without giving a full description.

The presence of singularities prevent stable and unstable curves to possess a lower bound for their size in any part of the phase-space. Therefore the product structure - the key ingredient of several hyperbolic argument - can only be introduced in a complicated set. 


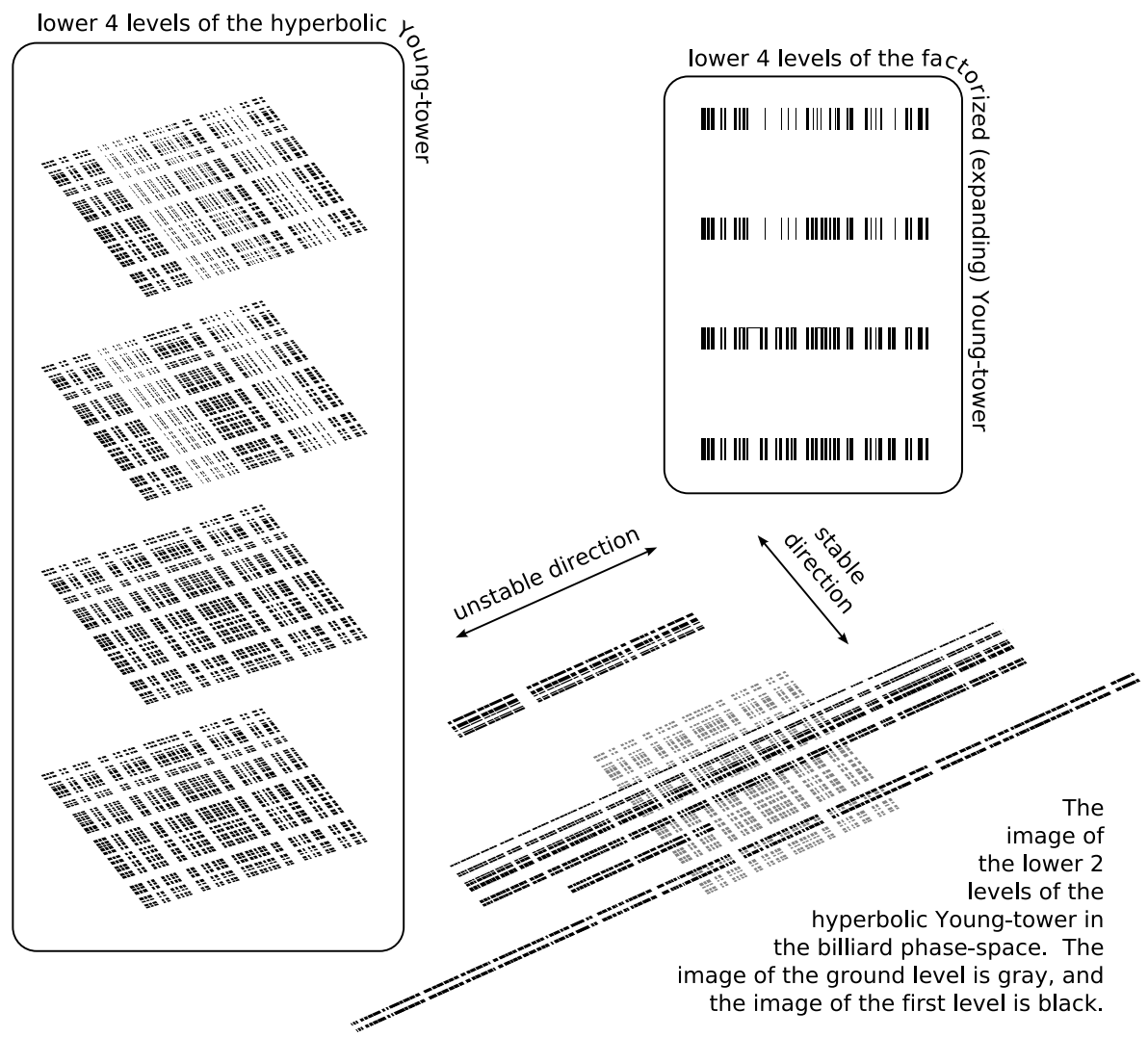

Figure 7: Young-towers, and Markov-return 
By the local ergodicity theorem for semi-dispersing billiards (cf. [Ch-S 87]) it is possible to choose an unstable curve $W$, which is short enough to ensure: a high amount of the points possesses unstable curve of this length. Then define a subset of this curve consisting of points, which remain a certain (exponentially shrinking) distance apart from $\mathcal{S}_{-1}$ and other more technical singularities.

$$
\Omega_{\infty}:=\left\{y \in W \mid d\left(T^{n} y, \mathcal{S}\right)>\delta_{1} \lambda^{-n} \quad \forall n \geq 0\right\}
$$

where $\lambda$ is the hyperbolicity constant. If $\delta_{1}$ is chosen small enough this set has positive measure. By construction each point in $\Omega_{\infty}$ possesses a stable curve of length $\delta_{1}$.

So far we have one unstable curve $W$, and a family of stable curves $\left\{\gamma^{s}\right\}$. Let us consider all the nearby unstable curves, which are long enough, and intersect all the stable curves in the previous family. These two families of curves $\left\{\gamma^{s}\right\}$ and $\left\{\gamma^{u}\right\}$ define the hyperbolic product-set $\Lambda=\left(\cup \gamma^{u}\right) \cap\left(\cup \gamma^{s}\right)$.

This set is the base of the hyperbolic Young-tower. To continue the construction of the tower we are going to focus on recurring subsets of $\Lambda$. On figure 7 we can see that some parts of $\Lambda$ are mapped to $\Lambda$. However we are only interested in those returns, which respect the product structure. A subset of $\Lambda$ is said to be an s-subset if it is the product of the full family of unstable curves and some part of the stable family. The notion of $u$-subset is defined -mutatis mutandis- in the same way.

We can see three intersections on the figure, the lower and upper ones are u-subsets. Talking about these intersections black covers grey in the unstable direction (when the reader sees black in these intersections, then on that unstable curve black covers grey). On the contrary grey covers black in the stable direction (on each stable line black can appear only where grey is already there). The inverse image of each of these two intersections is an s-subset.

A Markov-return is an event when some $T^{n} \Lambda \cap \Lambda$ is a u-subset, and it's inverse image under $T^{-n}$ is an s-subset. The possible non-Markov returns are when the intersection is not a u-subset (this is printed as the middle intersection), or when the inverse image is not an s-subset. This latter event occurs when a recurring part goes over the edge of $\Lambda$ in the stable direction.

The inverse image of the Markov-recurring part is not necessarily a solid rectangle intersected with $\Lambda$. It can have infinitely many "holes" in it, as demonstrated on figure 7 .

The tower is built using these Markov-type returns. The basic $\Lambda$ set is divided into s-subsets according to Markov-returns, and each subset is marked by the return time $R$. In this way $R$ will be a function on $\Lambda$ which is constant on these s-subsets. Not all Markov-returns are considered, for sophisticated details please consult [You 98]! The tower itself

$$
\Delta \stackrel{\text { def }}{=}\{(x, \omega): x \in \Lambda ; \omega=0,1, \ldots, R(x)-1\}
$$

and the dynamics on the tower is

$$
F(x, \omega)= \begin{cases}(x, \omega+1) & \text { if } \quad \omega+1<R(x) \\ \left(T^{R} x, 0\right) & \text { if } \quad \omega+1=R(x)\end{cases}
$$

Note that we have a decomposition into s-subsets which give rise to a Markov partition on the tower. This tower is only hyperbolic, and as a usual tool in this 
field Young has also introduced a factorised version of it $\bar{\Delta}$. Simply collapse the stable direction! This is also demonstrated on figure 7 . We have the following commutative diagram of measure preserving transformations:

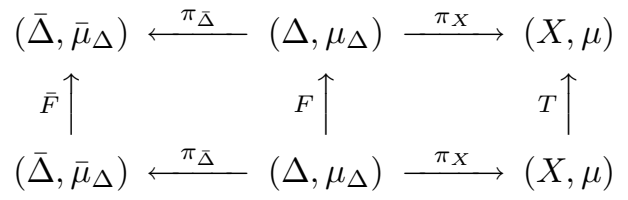

The projection to the original phase-space is not 1-1. On figure 7 the intersection in the middle has at least two inverse images. One of them is in the ground floor, and the other is on the first floor. Since the return is not Markovian these point are to be considered as different points on the tower.

Functions on the original phase space $X$ can be lifted to $\Delta$. Functions on $\Delta$ which are constant along stable directions can be considered as functions on $\bar{\Delta}$. For any function $\psi$ on $\Delta$ there exists functions $h$ and $\varphi$, such that $\varphi-\psi=h-h \circ F$, and $\varphi$ is constant along stable directions. In this equation the regularity of the functions can be examined, but we will skip the details, and only introduce distance, and function norms on the factorised tower $\bar{\Delta}$.

Remember that the factorised tower $\bar{\Delta}$ has a Cantor-structure, and a Markovpartition. The Cantor-hierarchy can be redefined with the separation time

$$
\begin{aligned}
& s(x, y)=\min \{k \geq 0 \mid \\
& \left.\quad \bar{F}^{k} x \text { and } \bar{F}^{k} y \text { lie in different elements of the Markov-partition }\right\} .
\end{aligned}
$$

With any $0<\beta<1$ the function $\beta^{s}$ is a metric providing the original Cantor topology.

On $\bar{\Delta}$ Young uses two kind of norms: the $\mathcal{C}$ norm is

$$
\|\varphi\|_{\mathcal{C}} \stackrel{\text { def }}{=} \sup _{l, j}\left\|\left.\varphi\right|_{\Delta_{l, j}}\right\|_{\infty} e^{-l \epsilon}
$$

where $\|\cdot\|_{\infty}$ is the essential supremum wrt $\mu$, and the indices $(l, j)$ refer to the elements of the Markov-partition. The $\mathcal{L}$ norm is a sum of this, and the $h$-norm:

$$
\|\varphi\|_{h} \stackrel{\text { def }}{=} \sup _{l, j}\left(\sup _{x, y \in \Delta_{l, j}} \frac{|\varphi(x)-\varphi(y)|}{\beta^{s(x, y)}}\right) e^{-l \epsilon}
$$

where the inner sup is again essential supremum wrt $\mu \times \mu$. For a Hölder function $f$ on the original billiard phase-space, we can associate a function on $\bar{\Delta}$ as described above, such that for any $\beta$ smaller than a certain number (computed from the original Hölder exponent of $f$ ) the resulting $\varphi$ has a finite $h$-norm.

In these definitions the role of $\epsilon$ is the following: without $\epsilon$ the Jacobian of the mapping would be 1 except when recurring to the base of the tower. However estimates expressed in the terms of this norm see a uniform expansion. Not to do harm the mapping should be expanding, when recurring to the base, so we have to choose $\epsilon$ smaller then the Lyapunov-exponent.

The Perron-Frobenius operator $P$ is defined on functions on $\bar{\Delta}$ with finite $\mathcal{L}$-norm as the adjoint of $\bar{F}$ wrt the measure $\bar{\mu}_{\Delta}$.

$$
\int f \cdot g \circ \bar{F} d \bar{\mu}_{\Delta}=\int P(f) \cdot g d \bar{\mu}_{\Delta} \quad f, g \in \mathcal{L}
$$


It also has an algebraic form

$$
P(f)(x)=\sum_{y \mid \vec{F} y=x} \frac{f(y)}{J(y)} \quad f \in \mathcal{L}
$$

where $J$ in the denominator is the Jacobian $\frac{\partial \bar{\mu}_{\Delta}^{*}}{\partial \bar{\mu}_{\Delta}}$ the Radon-Nikodym derivative of the pullback measure wrt the original measure. On the top of the tower the number of inverse images is infinite, otherwise there is a single preimage, and the Jacobian is 1 .

The main issue here is that the transfer operator $P$ possesses a spectral gap. This is the main reason for introducing this rather complicated and implicit symbolic dynamics. This gives those analytical tools in our hands which we describe now. Although $\kappa$ is defined in the original phase-space $X$, we will use the same notation for the function lifted to the tower. Since $\kappa$ is locally constant it can also be considered as a function on $\bar{\Delta}$.

\subsection{Fourier transform of the Perron-Frobenius operator}

In our case the Birkhoff sum $S_{n}=\sum_{k} \kappa \circ T^{k}$ is not an independent sum. Since Nagaev's 1957 work, [Nag 57], for concluding a limit theorem, one traditionally uses the Fourier-transform of the transfer operator. The Fourier transform operator is defined on the Young-tower, since that is the symbolic space, on which we have the full power of analytical tools built on the transfer operator:

$$
P_{t}(h) \stackrel{\text { def }}{=} P\left(e^{i\langle t, \kappa\rangle} h\right) \quad\left(h \in \mathcal{L}, t \in \mathbb{R}^{2}\right)
$$

It has the following simple connection with the characteristic function of the Birkhoff-sum:

$$
\int \exp \left(i\left\langle t, S_{n}\right\rangle\right) d \mu=\int P_{t}^{n}(1) d \bar{\mu}_{\Delta}
$$

For small values of $t, P_{t}$ can be considered as a perturbation of $P$, since $P_{0}=P$. Then one, in general, proves that $P_{t}$ possesses a gap between the leading simple eigenvalue $\lambda_{t}$ and the rest of the spectrum and the gap is uniformly bounded away from zero.

For large values of $t$ one needs to know exactly for which $t$ values will the unit circle intersect the spectrum. In our case for the continuous free flight function this occurs when $t \in 2 \pi \mathbb{Z}^{2}$. For that reason we switched to the discretised function $\kappa$, since the latter one is an integer valued vector function, and thus $P_{t}=P$ when $t \in 2 \pi \mathbb{Z}^{2}$, so we can factorise, and consider $t \in 2 \pi \mathbb{T}^{2}$. This is the question of minimality which is completely described in [SzV 04a].

Moreover, if our interest is a global limit law, then it is sufficient to derive an appropriate expansion for $\lambda_{t}$ for small values of $t$.

Indeed, this is also contained in the statement of Theorem 13 from [BG 06]. As a matter of fact, our main task will be to check the key condition of that theorem and thus we will actually derive a simple asymptotics $P_{t}^{n}=\lambda_{t}^{n}+O\left(\vartheta^{n}\right)$, for a suitable $\vartheta<1$. Consequently, the characteristic function of the dependent sum can be approximated with a power. Thus it is only the asymptotics of $\lambda_{t}$ that has remained to be investigated, and we expect the same behaviour what we have seen in the single term characteristic function. This will mean that the effect of dependence is negligible: both the independent and the dependent 
sums have the same asymptotic expansion for the Fourier transform, namely $1+\mathbf{c} n|t|^{2} \log |t|$. (In this formula $\mathbf{c}$ describes the direction-dependence, and $|t|$ the length, remember the definition of $\mathbf{c}$ in (1)!) Further, relying upon the method of $[\mathrm{SzV} 04 \mathrm{a}]$, we will also be able to handle the local version of the global limit law.

\section{Proof of Theorem 1: The asymptotics of $\lambda_{t}$}

In the forthcoming section we are going to establish the global limit law of Theorem 1 via an asymptotic expression at $t \rightarrow 0$ of $\lambda_{t}$, the leading eigenvalue of the Fourier-transform of the Perron-Frobenius operator. We will rely upon ideas of [BG 06] and of our work [SzV 04b].

The following theorem of [BG 06] provides a condition for a limit law in the non-standard domain of attraction of the Gaussian law for models possessing a Young tower with an exponential tail bound for the height function, in general.

Theorem 13. ([BG 06], Theorem 3.5) Assume that the distribution of a function $g \in \mathcal{L}$ is in the nonstandard domain of attraction of the normal law (wrt $\left.\bar{\mu}_{\Delta}\right)$. Remember that $\omega$ was the level function of the tower. Denote the "towersum" by $G=\sum_{k=1}^{\omega} g \circ \bar{F}^{-k}$. Let $L, l$ be as in subsection 2.5. Assume, moreover, that $l(x \log x) / l(x) \rightarrow 1, \quad L(x \log x) / L(x) \rightarrow 1$ when $x \rightarrow \infty$. Finally, assume that there exists a real number $a \neq-1 / 2$ such that

$$
\int g\left(e^{i t G}-1\right) d \bar{\mu}_{\Delta}=(a+o(1)) i t L(1 /|t|) \quad(t \rightarrow 0) .
$$

Write $L_{1}(x)=(2 a+1) L(x)$, and choose a sequence $B_{n} \rightarrow \infty$ such that

$\frac{n}{B_{n}^{2}} L_{1}\left(B_{n}\right) \rightarrow 1$. Then for $\lambda_{t}$ the leading eigenvalue of the $P_{t}(h)=P\left(e^{i t g} h\right)$

Fourier-transform-operator:

$$
\lambda_{t}=1-\frac{t^{2}}{2} L_{1}(1 /|t|)(1+o(1))
$$

and consequently for the $S_{n}=\sum_{k=0}^{n-1} g \circ \bar{F}^{k}$ Birkhoff-sum:

$$
\frac{S_{n}-n \int g}{B_{n}} \stackrel{\mathrm{d}}{\rightarrow} \mathcal{N}(0,1)
$$

We will indeed show that in our case the integral condition (3) of the above theorem holds for $g=\kappa$ with $a=0$. (This is actually the case discussed by [AD 01b].) We note that this case will allow a simpler treatment than that of the stadium because here the expansion rate is much larger (though the logarithm of the free flight function in a corridor does not form a random walk as it is the case in the stadium).

Our arguments for the local limit theorem will use again the asymptotics of the main eigenvalue $\lambda_{t}$, which is expressed for our case by the next Corollary.

Corollary 14. The Fourier transform operator of the discretised free-flight function has the following expansion for its leading eigenvalue:

$$
\lambda_{t} \sim 1+\mathbf{c} n|t|^{2} \log |t|
$$


Turn to the proof of the integral condition (3). In our case the function $\kappa$ is locally constant, so we can pass the integral immediately to $\Delta$. Denote the "tower-sum" by $K=\sum_{k=1}^{\omega} \kappa \circ F^{-k}$. It is easy to see that the dominating terms of the integral in (3) are those corresponding to parts of the phase space when the process is close to a singular point say $x_{0}$ (discussed in 2.2).

The estimate of this integral is based on the following fact. We have already observed that high values of $\kappa$ are typically reached rapidly: $\kappa \circ T^{-1}$ is in the order of $\sqrt{|\kappa|}$. During this fast trajectory segment the tower-sum can be estimated with the last term, hence it is also of order $\sqrt{|\kappa|}$. Since $\left|e^{i t \bar{K}}-1\right| \leq t \bar{K}$, the integral can be estimated by $t \sum_{n} n \sqrt{n} \cdot \mu\{|\kappa|=n\}=O(t)$. The trajectories which do not provide fast reach have polynomially small (in $|\kappa|$ ) relative measure in the level-sets. These domains can be discarded due to the following lemma:

Lemma 15. Any part of the integration domain $A$ with measure $\mu(A \cap\{|\kappa|=$ $N\})=O\left(N^{-3-\alpha}\right)$ with any $\alpha>0$ can be thrown away:

$$
\int_{\Delta} \kappa\left(e^{i\langle t, K\rangle}-1\right)=\int_{\Delta \backslash A} \kappa\left(e^{i\langle t, K\rangle}-1\right)+O(|t|)
$$

This is proved in proposition (4.17) of [BG 06]. Though the integration domains are in the hyperbolic Young-tower, if we identify the sets to be disregarded on the original phase-space $X$, then by measure preservation their pullbacks will satisfy the lemma.

\subsection{Reduction of the integration domain}

First we are going to discard that part $A_{1}$ of the neighbourhood of $x_{0}$ where the last step was not fast enough, i. e. $A_{1}=\left\{\left.x \in U_{0}|| \kappa \circ T^{-1}|>| \kappa\right|^{\frac{3}{4}}\right\} \subset X$. We also discard the set $A_{2}$ of those points where $\kappa \circ T^{-1}=w_{0}$, i. e. $A_{2}=U_{0} \backslash U_{0}^{\prime}$.

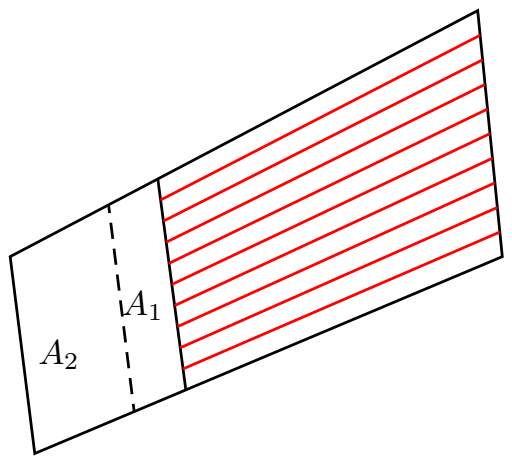

Figure 8: The discarded sets $A_{1}$ and $A_{2}$ in the level set of $\kappa$ and the foliation with nearly unstable curves

We already know that the relative measure of $\left(A_{1} \cup A_{2}\right)$ inside $\{|\kappa|=N\}$ is $O\left(N^{-1 / 2}\right)$ (cf. propositions 11 and 12).

Let us foliate the remaining part with curves whose direction is nearly unstable (the derivative is in the unstable cone for $T^{-1}$ ). 
The third discarded set $A_{3}$ will consist of points, for which the backward $V \log N$-step trajectory meets the $\left\{|\kappa|>N^{\frac{4}{5}}\right\}$ set, where $V$ is a large number to be chosen later. To estimate the relative measure of $A_{3}$ we prove the following lemma:

Lemma 16. There exists a constant $C$ such that, for any large enough integer $V$ it is true: for any large enough $N$, given any unstable curve $\mathcal{D}$ (for the mapping $T^{-1}$ ) in the set $\{|\kappa|=N\} \backslash \bigcup_{i=1,2} A_{i}$, the points for which $|\kappa|$ increases above $N^{4 / 5}$ within $V \log N$ iterations of $T^{-1}$ occupy a subset whose relative measure is less than $C N^{-1 / 40}$ in that curve.

Proof. The proof is a suitable modification of Lemma 4.18 of [BG 06]. We are going to apply the growth lemma of Chernov as formulated in [Ch 06] (lemma 2.2 ) (or in section 5.9 of [ChM 06]). The validity of this lemma in infinite horizon planar billiards was proved in [Ch 99]. We briefly recall the statement. The subject of the lemma is a possibly countable collection of smooth curves $\gamma$ which satisfies the following properties:

- the derivative is in the unstable cone (then the curves are called unstable ones),

- the components are contained in homogeneity strips (if the curve goes through the boundary of a homogeneity strip, then the intersection with the boundary is considered as a singularity point on the curve),

- and the lengths of the components do not exceed a fixed sufficintly small constant $\delta_{1}$.

The evolution of such a curve is that the mapping is applied to it. After that, the image is cut by boundaries of homogeneity strips. The resulting components which are longer than $\delta_{1}$ are cut into pieces of length between $\delta_{1} / 2$ and $\delta_{1}$. This way the image will satisfy the same properties.

The essence of the growth-lemma is the following. When applying the above procedure to $\gamma$ it is stretched and cut. The growth lemma bounds the total measure of points close to the boundaries of these pieces, or in other words it provides a lower bound for the total measure of sufficiently long pieces.

Lemma 17. (Growth Lemma 2.2, [Ch 06]). There exists positive constants $\delta_{1}, c_{1}, c_{2}$, and $\theta_{1}<1$ and $\lambda>1$, such that if $\gamma$ satisfies the above properties, then for any $n \geq 0$ and $\epsilon>0$ we have

$$
\operatorname{Leb}_{\gamma}\left(r_{n}(x)<\epsilon\right) \leq c_{1}\left(\theta_{1} \lambda\right)^{n} \operatorname{Leb}_{\gamma}\left(r_{0}(x)<\epsilon / \lambda^{n}\right)+c_{2} \epsilon \operatorname{Leb}_{\gamma}(\gamma)
$$

where $r_{n}$ means distance from the boundary in the $n^{\text {th }}$ image (i. e. that of the point $T^{n} x$ from the boundary of the component of $T^{n} \gamma$ which contains it.

We are going to apply the lemma to the mapping $T^{-1}$ and to the image of the curve $\mathcal{D}$ under the above procedure (apply $T^{-1}$ and cut)! We note that distances, and $\mathrm{Leb}_{\gamma}$ is necessarily meant in the original coordinates, rather than the new ones. Within $|\kappa|=N^{4 / 5}$ the largest homogeneity strip has index $c N^{1 / 5}$ and width $c N^{-3 / 5}$. Hence the points which reach $|\kappa|>N^{4 / 5}$ will sit on a curve component with length not larger than $c N^{-3 / 5}$. We are going to estimate the relative measure of points of the latter type using the growth lemma for all $n<V \log N$ and for $\epsilon=c N^{-3 / 5}$. 
Note that in the set $\{|\kappa|=N\} \backslash A_{1}$ one has $\left|\kappa \circ T^{-1}\right|<N^{\frac{3}{4}}$. Hence the image will intersect $\{|\kappa|=M\}$ sets with $c N^{1 / 2}<M<N^{3 / 4}$. We are going to estimate the relative measure in a $\{|\kappa|=M\}$ set:

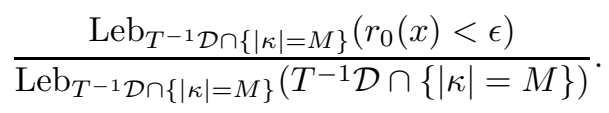

Let us first investigate $M=N^{3 / 4}$. The set in the numerator will contain the intersection of the curve with the union of those homogeneity strips, for which the width is smaller than $\epsilon=c N^{-3 / 5}$. That is $k^{-2}$ for the smallest index $k$ for which $k^{-3}<\epsilon$. This set has measure $c N^{-2 / 5}$. For each of the remaining $k$ indices we have $2 \epsilon$. The number of the remaining indices is $c N^{3 / 16}-c N^{1 / 5}$, so the contribution to the measure is $c N^{-33 / 80}$. Therefore the numerator is $c N^{-2 / 5}$. The denominator is $c M^{-1 / 2}=c N^{-3 / 8}$ if the curve has a complete intersection with $\{|\kappa|=M\}$. In that case the relative measure is $c N^{-1 / 40}$. For other $M$ values the relative measure is even smaller. Hence if an intersection is not complete it continues in an other $\{|\kappa|=M\}$ domain, and for the union of these pieces we have the same relative measure estimate. So we have the same relative measure for the whole $T^{-1} \mathcal{D}$ except possibly for the neighbourhood of the endpoints $T^{-1} \partial \mathcal{D}$. There are two of them and the neighbourhood has relative measure at most $c N^{-1}$ inside $\mathcal{D}$.

Now we apply the growth lemma:

$$
\frac{\operatorname{Leb}_{\mathcal{D}}\left(r_{n}(x)<\epsilon\right)}{\operatorname{Leb}_{\mathcal{D}}(\mathcal{D})} \leq c_{1}\left(\theta_{1} \lambda\right)^{n-1} \frac{\operatorname{Leb}_{\mathcal{D}}\left(r_{1}(x)<\epsilon / \lambda^{n-1}\right)}{\operatorname{Leb}_{\mathcal{D}}(\mathcal{D})}+c_{2} \epsilon
$$

The first term on the right hand side can be bounded by elements of a geometric series, so if we sum for $n<V \log N$ it will be proportional to the first term which is $c N^{-1 / 40}$ from the above discussion. The second term gives a sum $c V(\log N) N^{-3 / 5}$.

The last discarded set will be defined on the tower: We are going to throw away that part $A_{4}$ of the integration domain, which is too high on the tower $\omega>V \log |\kappa|$. Since the tower has exponentially small tails, if $V$ was chosen large enough, then the discarded set has measure $\mu_{\Delta}\left(A_{4} \cap\{|\kappa|=N\}\right)=O\left(N^{-4}\right)$, and thus by lemma 15 the integral can be restricted to its complement.

Now it remained to estimate the integral on the non-discarded set.

\section{Lemma 18.}

$$
\int_{\pi_{X}^{-1}\left(X \backslash \bigcup_{i=1}^{3} A_{i}\right) \backslash A_{4}} \kappa\left(e^{i\langle t, K\rangle}-1\right)=O(|t|)
$$

Proof. Consider the integral on the left. By the definition of the discarded sets we have:

$$
\begin{aligned}
& \left|\int_{\pi_{X}^{-1}\left(X \backslash \bigcup_{i=1}^{3} A_{i}\right) \backslash A_{4}} \kappa\left(e^{i\langle t, K\rangle}-1\right)\right| \leq \\
& \quad \leq|t| \int_{\pi_{X}^{-1}\left(X \backslash \bigcup_{i=1}^{3} A_{i}\right) \backslash A_{4}}|\kappa||K| \leq C|t| \sum_{n} \mu\{|\kappa|=n\} n \log n n^{4 / 5} \leq C|t|
\end{aligned}
$$




\section{Proof of Theorems 2, 3 and 4}

The proof of the local limit theorems (Theorems 20 and 21 below) and that of the recurrence is -mutatis mutandis- the same as for the finite horizon case [SzV 04a]. Here we are going to state the theorems only, and comment just the beginning, where the different norms used by the different approaches ([BG 06] and $[\mathrm{SzV}$ 04a]) could lead to a confusion.

Theorem 19 (Nagaev-type theorem, modification of [SzV 04a] Theorem 3.3). There are constants $\epsilon>0, K>0$ and $\theta<1$ and a function $\rho:(-\epsilon, \epsilon) \rightarrow \mathcal{L}$ such that

$$
\left\|P_{t}^{n} h-\lambda_{t}^{n} \rho_{t} \int_{\bar{\Delta}} h d \bar{\mu}_{\Delta}\right\|_{L^{1}} \leq K \theta^{n}\|h\|_{\mathcal{L}} \quad \forall|t|<\epsilon, n \geq 1, h \in \mathcal{L}
$$

and

$$
\left\|\rho_{t}-1\right\|_{L^{1}} \rightarrow 0(t \rightarrow 0), \quad \lambda_{t}=1+(1+o(1)) \mathbf{c}|t|^{2} \log |t|
$$

The statement in [SzV 04a] was stronger in the sense of norms, but inequality (4) is completely enough for the proof of our local limit theorems. The proof of Theorem 19 can also be straightforwardly derived from considerations in [BG 06], namely the proof of their theorem 3.5.

Theorem 20 (Local limit theorem). Suppose that the corridor free flights $\{\kappa(x) \mid x \in \partial X, T x=x\}$ span the plane. Let $k_{n} \in \mathbb{Z}^{2}$ be such that $\frac{k_{n}}{\sqrt{n \log n}} \rightarrow$ $k \in \mathbb{R}^{2}$. Then

$$
n \log n \mu\left\{S_{n}=k_{n}\right\} \rightarrow \varphi(k)
$$

where $\varphi$ is a non-degenerate normal density function with zero expectation and covariance matrix

$$
\sum_{x \in \partial X \mid T x=x} \frac{c_{\mu} d_{x}^{2}}{2|\kappa(x)|}\left(\begin{array}{cc}
\kappa_{1}^{2}(x) & \kappa_{1}(x) \kappa_{2}(x) \\
\kappa_{1}(x) \kappa_{2}(x) & \kappa_{2}^{2}(x)
\end{array}\right)
$$

where $\kappa=\left(\kappa_{1}, \kappa_{2}\right)$ is the notation for the component functions.

The proof of recurrence follows a version of the Borel-Cantelli lemma due to Lamperti. See [SzV 04a] for the statement, conditions and the details of application. For this part of the Borel-Cantelli lemma one needs some kind of independence. Lamperti's condition can be fulfilled when one proves an asymptotic independence statement:

Theorem 21. Let $j_{n} \in \mathbb{Z}^{2}$ be such that $\frac{j_{n}}{\sqrt{n \log n}} \rightarrow j \in \mathbb{R}^{2}$, and $k_{n} \in \mathbb{Z}^{2}$ be such that $\frac{k_{n}}{\sqrt{n \log n}} \rightarrow k \in \mathbb{R}^{2}$. If the corridor free flights span the plane, then

$\lim _{m, n-m \rightarrow \infty} m \log m(n-m) \log (n-m) \mu\left\{S_{m}=j_{m}, S_{n}=j_{m}+k_{n-m}\right\}=\varphi(j) \varphi(k)$.

The proof consists of repeating twice the same integral transformations as in the proof of the local limit theorem (again see the proof of Theorem 4.2 in [SzV 04a] for details; we note, however, that the claim of Theorem 4.2 in [SzV 04a] contains a misprint, in fact it ought to be analogous with that of Theorem 21). 
Theorem 22. The invariant (infinite) measure of the Lorentz process $d \tilde{\mu}=$ $\langle v, n(q)\rangle d q d v$ (where $d q$ is the arclength measure on the boundary of infinitely many scatterers) is ergodic $i$. e. for any invariant set $A$ either $\tilde{\mu}(A)=0$ or $\tilde{\mu}(\tilde{X} \backslash A)=0$.

This follows from recurrence and [Sim 89], where the author showed the equivalence between recurrence and the ergodicity of the infinite measure (see also [Pen 00]).

Acknowledgement. The authors express their sincere thanks to Péter Bálint and Sebastien Gouëzel for making the first versions of their paper [BG 06] available in statu nascendi. They are also most grateful to the referee for her/his valuable remarks.

\section{References}

[AD 01a] J. Aaronson and M. Denker. Local limit theorems for partial sums of stationary sequences generated by Gibbs-Markov maps. Stochastic Dynamics 1(2):193-237, 2001.

[AD 01b] J. Aaronson and M. Denker. A local limit theorem for stationary processes in the domain of attraction of a normal distribution. In N. Balakrishnan, I. A. Ibragimov, and V. B. Nevzorov, editors, Asymptotic methods in probability and statistics with applications. Papers form the interbational conference, St. Petersburg, Russia, 1998, pages 215-224, Birkhäuser, 2001

[BG 06] P. Bálint and S. Gouëzel. Limit theorems in the stadium biliard. Commun. Math. Phys. 263:461-512, 2006

[B 92] P. M. Bleher. Statistical Properties of Two-Dimensional Periodic Lorentz Gas with Infinite Horizon. J. of Stat. Physics 66(1):315373, 1992 .

[BD 85] J.-P. Bouchaud and P. Le Doussal. Numerical study of the Ddimensional periodic Lorentz gas with universal properties. J. Stat. Phys. 41:225-248, 1985.

[Bun 85] L. A. Bunimovich. Decay of correlations in dynamical systems with chaotic behaviour. Zh. Eksp. Teor. Fiz. 89:1452-1471, 1985 [Sov. Phys. JETP 62:842-852, 1985].

[BChS 91] L. A. Bunimovich and N. I. Chernov and Ya. G. Sinai. Statistical properties of two-dimensional hyperbolic billiards. Russ. Math. Surveys 46:47-10, 1991.

[BS 80] L. A. Bunimovich and Ya. G. Sinai. Markov partitions for dispersed billiards. Comm. Math. Phys. 73:247-280, 1980.

[BS 81] L. A. Bunimovich and Ya. G. Sinai. Statistical properties of Lorentz gas with periodic configuration of scatterers. Comm. Math. Phys. 78:479-497, 1981. 
[Ch 99] N. I. Chernov. Decay of correlations and dispersing billiards. Journal of Statistical Physics 94:513-556, 1999.

[Ch 06] N. I. Chernov. Advanced statistical properties of dispersing billiards. Journal of Statistical Physics 122:1061-1094, 2006.

[ChM 06] N. I. Chernov and R. Markarian. Chaotic Billiards. AMS Math. Surveys, Vol. 127. 2006.

[Ch-S 87] N. I. Chernov and Ya. G. Sinai. Ergodic properties of some systems of two-dimensional disks and three-dimensional balls. (Russian) $\mathrm{Us}$ pekhi Mat. Nauk 42, no. 3(255), 153-174, 256, 1987.

[Con 99] J.-P. Conze. Sur un critère de rcurrence en dimension 2 pour les marches stationnaires, applications. Ergodic Theory and Dynamical Systems 19(5):1233-1245, 1999.

[F 66] W. Feller. An Introduction to Probability Theory and its Applications. Volume II. Wiley and Sons, 1966

[FM 84] B. Friedman and R. F. Martin, Jr. Decay of the velocity autocorrelation function for the periodic Lorentz gas. Phys. Lett. 105A:23-26, 1984.

[GH 88] Y. Guivarc'h and J. Hardy. Théorèmes limites pour une classe de chaînes de Markov et applications aux difféomorphismes d'Anosov. Ann. Inst. Henri Poincaré 24(1):73-98, 1988.

[IL 71] I. A. Ibragimov and Yu. V. Linnik. Independent and Stationary Sequences of Random Variables, Nauka, Moscow, 1971.

[KSSz 90] A. Krámli and N. Simányi and D. Szász. A "transversal" fundamental theorem for semi-dispersing billiards. Comm. Math. Phys. 129:535-560, 1990.

[KSz 83] A. Krámli and D. Szász. Random walks with internal degrees of freedom. I. Local limit theorems. Z. Wahrscheinlichkeitstheorie 63:8595, 1983.

[KSz 85] A. Krámli and D. Szász. The Problem of Recurrence for Lorentz Processes. Comm. in Math. Phys. 98:539-552, 1985.

[L 03] M. Lenci, Aperiodic Lorentz gas:recurrence and ergodicity, Ergodic Theory Dynam. Systems 23:869-883, 2003

[Nag 57] S. V. Nagaev. Some limit theorems for stationary Markov chains. Theor. Probab. Appl. 2:378-406, 1957.

[Pen 00] F. Pène. Applications des propriétés stochastiques du billiard dispersif. C. R. Acad. Sci. Paris Sér. I Math. 330:1103-1106, 2000.

[Sch 98] K. Schmidt. On joint recurrence. C. R. Acad. Sci. Paris Sr. 1 Math 327(9):837-842, 1998.

[Sim 89] N. Simányi. Toward a proof of recurrence for the Lorentz process Banach Center Publications, PWN, Warsaw 23:265-276, 1989. 
[Spi 64] F. Spitzer. Principles of random walks. chap. 6 26. Prop 3. Van Nostrand, 1964.

[SzV 04a] D. Szász and T. Varjú. Local limit theorem for the Lorentz process and its recurrence in the plane Ergodic Theory and Dynamical Systems 24:257-278, 2004.

[SzV 04b] D. Szász and T. Varjú. Markov Towers and Stochastic Properties of Billiards Modern dynamical systems and applications 433-445, Cambridge-Univ. Press, Cambridge 2004.

[You 98] L.-S. Young. Statistical properties of systems with some hyperbolicity including certain billiards. Ann. of Math. 147:585-650, 1998.

[ZGNR 86] A. Zacherl and T. Geisel and J. Nierwetberg and G. Radons. Power spectra for anomalous diffusion in the extended billiard. Phys. Lett 114A:317-321, 1986.

Domokos Szász, Tamás Varjú

Budapest University of Technology, Mathematical Intitute.

Budapest, Egry J. u. 1 Hungary H-1111

szasz@math.bme.hu.hu, kanya@math.bme.hu 